Wright State University

CORE Scholar

Physics Faculty Publications

Physics

10-1-2000

\title{
Electron Beam and Optical Depth Profiling of Quasibulk GaN
}

L. Chernyak

A. Osinsky

G. Nootz

A. Schulte

J. Jasinski

See next page for additional authors

Follow this and additional works at: https://corescholar.libraries.wright.edu/physics

Part of the Physics Commons

\section{Repository Citation}

Chernyak, L., Osinsky, A., Nootz, G., Schulte, A., Jasinski, J., Benamara, M., Liliental-Weber, Z., Look, D. C., \& Molnar, R. J. (2000). Electron Beam and Optical Depth Profiling of Quasibulk GaN. Applied Physics Letters, 77 (17), 2695-2697.

https://corescholar.libraries.wright.edu/physics/64

This Article is brought to you for free and open access by the Physics at CORE Scholar. It has been accepted for inclusion in Physics Faculty Publications by an authorized administrator of CORE Scholar. For more information, please contact library-corescholar@wright.edu. 


\section{Authors}

L. Chernyak, A. Osinsky, G. Nootz, A. Schulte, J. Jasinski, M. Benamara, Z. Liliental-Weber, David C. Look, and Richard J. Molnar

This article is available at CORE Scholar: https://corescholar.libraries.wright.edu/physics/64 


\title{
Electron beam and optical depth profiling of quasibulk GaN
}

\author{
L. Chernyak ${ }^{\mathrm{a})}$ \\ Physics Department, University of Central Florida, Orlando, Florida 32816-2385
}

A. Osinsky

Corning Applied Technologies, Woburn, Massachusetts 01801

G. Nootz and A. Schulte

Physics Department, University of Central Florida, Orlando, Florida 32816-2385

J. Jasinski, ${ }^{\text {b) }}$ M. Benamara, and Z. Liliental-Weber

Lawrence Berkeley National Laboratory, Berkeley, California 94720

D. C. Look

Wright State University and Air Force Research Laboratory, Dayton, Ohio 45435

R. J. Molnar

Massachusetts Institute of Technology, Lincoln Laboratory, Lexington, Massachusetts 02173-9108

(Received 28 July 2000; accepted for publication 28 August 2000)

\begin{abstract}
Electron beam and optical depth profiling of thick (5.5-64 $\mu \mathrm{m})$ quasibulk $n$-type GaN samples, grown by hydride vapor-phase epitaxy, were carried out using electron beam induced current (EBIC), microphotoluminescence (PL), and transmission electron microscopy (TEM). The minority carrier diffusion length, $L$, was found to increase linearly from $0.25 \mu \mathrm{m}$, at a distance of about $5 \mu \mathrm{m}$ from the GaN/sapphire interface, to $0.63 \mu \mathrm{m}$ at the GaN surface, for a 36- $\mu \mathrm{m}$-thick sample. The increase in $L$ was accompanied by a corresponding increase in PL band-to-band radiative transition intensity as a function of distance from the GaN/sapphire interface. We attribute the latter changes in PL intensity and minority carrier diffusion length to a reduced carrier mobility and lifetime at the interface, due to scattering at threading dislocations. The results of EBIC and PL measurements are in good agreement with the values for dislocation density obtained using TEM. (C) 2000 American Institute of Physics. [S0003-6951(00)00643-4]
\end{abstract}

As the III-V nitride technology becomes more and more mature, the necessity for a homoepitaxial substrate for nitride-based device development has become apparent. Homoepitaxial growth of GaN has proven its tremendous potential to achieve superior material quality resulting in extremely narrow photoluminescence linewidths ${ }^{1}$ and a reduction of the dislocation density by six orders of magnitude. These material qualities can only be attained by using a substrate which is identical in crystal structure, lattice parameter, and thermal expansion coefficient. Due to limitations of the bulk-growth techniques at very high pressure $(\sim 15 \mathrm{kbar})$, a number of researchers have pursued hydride vapor-phase epitaxy (HVPE) as a quasibulk technique for the growth of thick ( $\geqslant 20 \mu \mathrm{m})$, large-area substrates or buffers. ${ }^{2,3}$ The thick layer growth is facilitated by the high growth rates $(\sim 1 \mu \mathrm{m} /$ min) and near-equilibrium nature of the process, which can be exploited to generate low-defect material. This letter reports on the correlation between optical and transport properties of thick HVPE quasibulk $n$-GaN samples with their microstructure.

The samples under investigation were grown at the MIT Lincoln Laboratory. The details of HVPE growth are reported elsewhere. ${ }^{2}$ Several different samples $\sim 5.5,36,55$, and $64 \mu \mathrm{m}$ thick were used in our research. Room-

${ }^{a)}$ Electronic mail: chernyak@physics.ucf.edu

${ }^{b)}$ Also at: Institute for Experimental Physics, Warsaw University, 00681 Warsaw, Poland. temperature Hall measurements showed $n$-type electrical conductivity for these samples, with electron concentrations (at the surface) ranging from $8 \times 10^{16}$ to $1.2 \times 10^{17} \mathrm{~cm}^{-3}$, and mobilities of $580-750 \mathrm{~cm}^{2} / \mathrm{V} \mathrm{s}$, respectively. However, each sample had a thin $(\sim 200 \mathrm{~nm})$, conductive interface layer with an electron concentration of mid $-10^{19} \mathrm{~cm}^{-3}$ and a mobility of about $50 \mathrm{~cm}^{2} / \mathrm{V} \mathrm{s}$. This interface layer greatly affected the overall electrical properties, and is discussed in more detail elsewhere. ${ }^{4}$

Electron beam induced current (EBIC) measurements were carried out in situ in a scanning electron microscope (SEM) JEOL 6400F. The accelerating voltage, used in these measurements, was varied from 10 to $20 \mathrm{kV}$. This corresponds to an electron range, $R$, of $0.36-1.20 \mu \mathrm{m}$, respectively. ${ }^{5}$ After cleavage, vertical gold (Au) stripes (Schottky barriers) of different sizes were formed on one of the edges of the samples by Au evaporation and subsequent lift off. The minority hole diffusion length, $L$, was derived from the line-scan EBIC measurements. ${ }^{6}$ The measurements were carried out at variable distance, $d$, from the GaN/ sapphire interface. In these measurements the electron beam (positioned perpendicular to the sample edge) was moved from the vertical wall of the $\mathrm{Au} / n$-GaN Schottky barrier toward another Au contact. $L$ can be obtained from the EBIC decay versus distance from the edge of the Schottky contact (for distances $>2 L$ ). We reported the details of the diffusion length extraction in Ref. 6. There was no need for sample thickness correction in the $L$ measurements, since the 


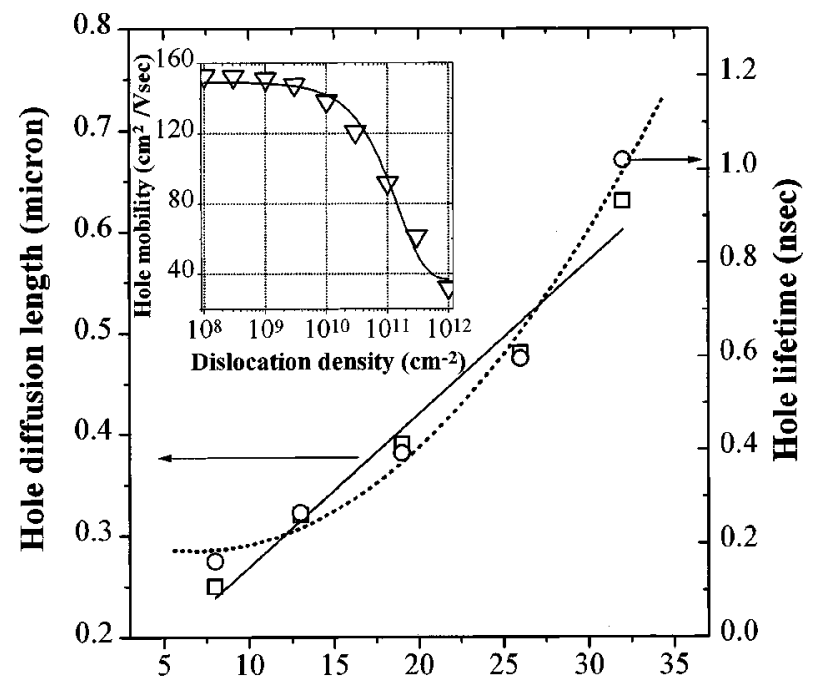

Distance from GaN/sapphire interface $(\mu \mathrm{m})$

FIG. 1. Experimental minority hole diffusion length dependence (open squares) on the distance, $d$, from the $n$-GaN/sapphire interface, measured for a 36- $\mu$ m-thick sample. The solid line shows a fit. The open circles show the calculated values of minority hole lifetime, $\tau$, using Eq. (1). The dashed line represents a second-order polynomial fit [cf. Eq. (1)]. Inset: Theoretical minority hole mobility dependence on dislocation density.

samples were considered as bulk material (thickness $\gg 4 L$ ). ${ }^{7}$ There were no EBIC resolution limitations as well, since the ratio $R / L \leqslant 4 .^{8}$

Micro-PL profiling across thick GaN samples was performed in situ in the microphotoluminescence setup. The micro-PL measurements were carried out using a $4 \mathrm{~ns}$ pulse $337 \mathrm{~nm}$ nitrogen laser with a power of $<100 \mu \mathrm{J} / \mathrm{pulse}$. The laser beam, focused in a 1.5 - $\mu \mathrm{m}$-diam spot, allowed microprobing of a region comparable to that studied by the EBIC technique (electron beam line-scan length is $\sim 4.4 \mu \mathrm{m}$ at $\times 25000$ magnification). A translation stage with $0.5 \mu \mathrm{m}$ step resolution was used for laser beam positioning at the predetermined distance from the GaN/sapphire interface.

Transmission electron microscopy (TEM) was applied to determine a depth profile distribution of threading dislocations present in the HVPE GaN layers. Several layers, with thicknesses of either $\sim 5.5$ or $\sim 55 \mu \mathrm{m}$, were chosen for this study. Both cross-sectional and plan-view TEM specimens were prepared by a standard method of mechanical prethinning followed by Ar-ion milling down to electron transparency. Plan-view samples were thinned from the substrate such that the region near the top surface of each layer could be examined. TEM studies were carried out using TOPCON 002B and JEOL 200CX microscope, operated at $200 \mathrm{kV}$. Bright field TEM images, recorded under multibeam conditions (in order to image all dislocations with different Burgers vectors), were used to estimate a density of dislocations.

The open squares in Fig. 1 represent the experimental dependence of minority carrier diffusion length on distance from the GaN/sapphire interface for one of the samples under investigation (36 $\mu \mathrm{m}$ thick). $L$ increases linearly from $\sim 0.25 \mu \mathrm{m}$ at the GaN/sapphire interface up to $\sim 0.63 \mu \mathrm{m}$ at the surface. This increase is in agreement with the average space between dislocations, calculated from the experimentally determined dislocation densities using TEM, and shown in Fig. 2. The dislocation density was measured for each

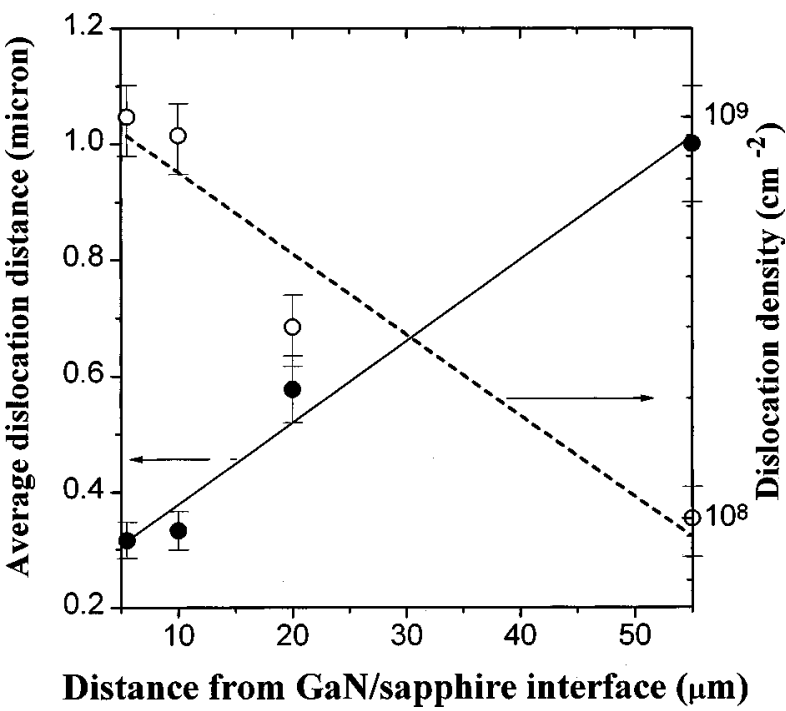

FIG. 2. Experimental dependence of threading dislocation density vs distance from the GaN/sapphire interface (open circles). The measurement error range is illustrated by the horizontal bars. The dashed line represents the fit. Also shown is average dislocation spacing (closed circles), calculated from the experimentally determined dislocation density. The linear fit is shown by the solid line.

sample under investigation at a few distances from the GaN/ sapphire interface. In such a way the experimental dependence, presented in Fig. 2, was obtained.

Comparing the dislocation densities in Fig. 2 with the $L$ vs $d$ dependence in Fig. 1, one can conclude that a decrease in minority carrier diffusion length toward the GaN/sapphire interface is related to an increase of dislocation density. The minority carrier diffusion length at a certain distance from the interface, therefore, can be related to the spacing between two adjacent dislocations. For example, $L=0.63 \mu \mathrm{m}$ at a distance of $32 \mu \mathrm{m}$ from the GaN/sapphire interface (cf. Fig. 1). From Fig. 2, the average spacing between two adjacent dislocations at this distance is $\sim 0.69 \mu \mathrm{m}$. Similarly, $L$ $=0.25 \mu \mathrm{m}$ at a distance of $\sim 8 \mu \mathrm{m}$ from the GaN/sapphire interface, while the spacing between two adjacent dislocations at this distance is $\sim 0.35 \mu \mathrm{m}$. Hence, it is likely that the minority carrier diffusion length is determined by carrier recombination on the adjacent threading dislocations.

The hole diffusion length in $n$-GaN may decrease due to a decrease in lifetime, $\tau$, and/or diffusivity (mobility). That is

$$
L=\sqrt{D \tau},
$$

and the diffusivity, $D$, and mobility, $\mu_{p}$, are related via the Einstein equation.

The calculation of hole mobility, $\mu_{p}$, is a difficult problem in any semiconductor material because of the degenerate valence bands. However, to get a rough estimate of $\mu_{p}$ versus dislocation density, we simply assumed a single valence band, with an associated hole effective mass of $2.0 m_{0}$, and then followed the treatment of Ref. 9. The results are presented in the inset of Fig. 1. Note that the value of mobility at low dislocation densities, $\sim 150 \mathrm{~cm}^{2} / \mathrm{V} \mathrm{s}$, is much higher than that found in most p-type GaN samples. However, such samples are usually heavily doped (with $\mathrm{Mg}$ ), and heavily compensated (probably with the $\mathrm{N}$ vacancy), and thus may be expected to produce a much lower mobility than that calculated for the excellent $n$-type GaN studied here. 


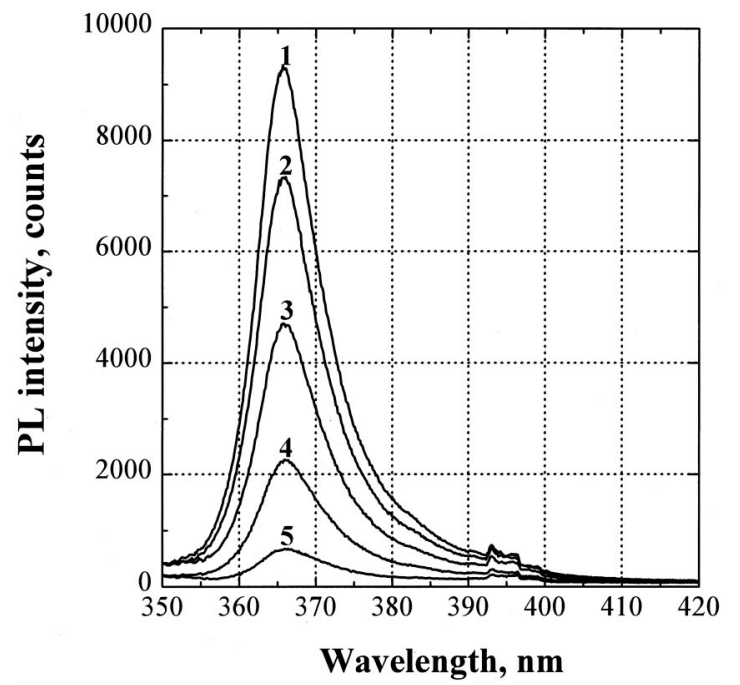

FIG. 3. Microphotoluminescence spectra measured across a $64-\mu \mathrm{m}$-thick sample using a $337 \mathrm{~nm} 4 \mathrm{~ns}$ pulse nitrogen laser beam, focused down to $\sim 1.5 \mu \mathrm{m}$. Spectrum 1 was measured at the sample surface, while spectrum 5 was measured at the GaN/sapphire interface.

We also found experimental evidence for the dependence of $\tau$ on $d$. Figure 3 shows PL spectra measured as a function of distance from the GaN/sapphire interface for a $64-\mu \mathrm{m}$-thick sample. One can see from Fig. 3 that the intensity of the room temperature band-to-band transition decreases as the probe moves closer to the GaN/sapphire interface. Since the PL intensity is proportional to the carrier lifetime,${ }^{10}$ we conclude that the latter parameter decreases at lower values of $d$.

Using Eq. (1), we can now find the minority hole lifetime dependence on distance from the GaN/sapphire interface, based on the experimental dependence of $L$ vs $d$ (cf. Fig. 1) and $\mu_{p}$ vs dislocation density (cf. inset of Fig. 1). The calculated values of $\tau$ vs $d$ are also presented in Fig. 1. To find the dislocation density corresponding to a certain distance from the GaN/sapphire interface, at which the $L$ measurements were carried out, we used the dislocation density versus $d$ data, presented in Fig. 2 . Since $L$ depends linearly on $d$ and on the square root of $\tau$ [cf. Eq. (1)], a quadratic fit for $\tau$ vs $d$ is understandable (see the dashed line in Fig. 1). We note the values of $\tau$, calculated in this work, are in agreement with the minority carrier lifetimes (1.4-2.3 ns), probed in InGaN/GaN quantum well, in situ in SEM, using time resolved cathodoluminescence. ${ }^{11}$
In summary, the minority hole diffusion length in thick HVPE-grown GaN samples has been measured as a function of distance from the GaN/sapphire interface. We found a linear increase of $L$ with increasing distance, which is in good agreement with an increase of the average distance between dislocations. We attribute the low values of minority carrier diffusion length at the GaN/sapphire interface to carrier recombination on the threading dislocations. This interpretation is reasonable, since the $L$ values are comparable to the adjacent dislocation spacing. The minority hole lifetime dependence as a function of sample thickness was calculated based on the experimentally measured $L$ values and the calculated mobility dependence on dislocation density.

L.C. and A.O. thank the members of the Witt-Wood initiative group for stimulating discussions. This work was supported in part by BMDO STTR Contract No. N0014-99-M0277, managed by ONR (program monitor Dr. J.C. Zolper) and Air Force Office of Scientific Research, through the U.S. Department of Energy under Order No. AFOSR-ISSA-000011. J.J., M.B., and Z.L.W. thank NCEM in Berkeley for the use of the TEM facility and W. Swider for TEM sample preparation. D.C.L. was supported by Air Force Contract No. F33615-00-C-5402 and Navy Contract No. N0001499-11067.

${ }^{1}$ M. Kamp, C. Kirchner, V. Schwegler, A. Pelzmann, K. J. Ebeling, M. Leszczynski, I. Grzegory, T. Suski, and S. Porowski, MRS Internet J. Nitride Semicond. Res. 4S1, G10 (1999).

${ }^{2}$ R. J. Molnar, W. Götz, L. T. Romano, and N. M. Johnson, J. Cryst. Growth 178, 147 (1997).

${ }^{3}$ T. Detchprohm, K. Hiramatsu, H. Amano, and I. Akasaki, Appl. Phys. Lett. 61, 2688 (1992).

${ }^{4}$ D. C. Look and R. J. Molnar, Appl. Phys. Lett. 70, 3377 (1997).

${ }^{5}$ Z. Z. Bandić, P. M. Bridger, E. C. Piquette, and T. C. McGill, Solid-State Electron. 44, 221 (2000); H. J. Leamy, J. Appl. Phys. 53, R51 (1982)

${ }^{6}$ L. Chernyak, A. Osinsky, H. Temkin, J. W. Yang, Q. Chen, and M. A. Khan, Appl. Phys. Lett. 69, 2531 (1996); L. Chernyak, A. Osinsky, V. Fuflyigin, and E. F. Schubert, ibid. 77, 875 (2000).

${ }^{7}$ C. A. Dimitriadis, J. Phys. D 14, 2269 (1981).

${ }^{8}$ K. L. Luke, O. von Roos, and L.-J. Cheng, J. Appl. Phys. 57, 1978 (1985).

${ }^{9}$ D. C. Look and J. R. Sizelove, Phys. Rev. Lett. 82, 1237 (1999).

${ }^{10}$ M. E. Aumer, S. F. LeBoeuf, M. Smith, L. Y. Lin, H. X. Jiang, and S. M. Bedair, Electronic Materials Conference, University of Denver, Denver, CO, 21-23 June, 2000 (unpublished)

${ }^{11}$ X. Zhang, D. H. Rich, J. T. Kobayashi, N. P. Kobayashi, and P. D. Dapkus, Appl. Phys. Lett. 73, 1430 (1998). 